\title{
Dynamics on the unit disk: Short geodesics and simple cycles
}

Curtis T. McMullen*

Mathematics Subject Classification (2010). 30D05, 37E45.

Keywords. Blaschke products, complex dynamics, rotation numbers.

\section{Contents}

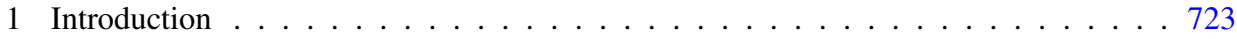

2 Simple cycles . . . . . . . . . . . . . . . . . . . . 727

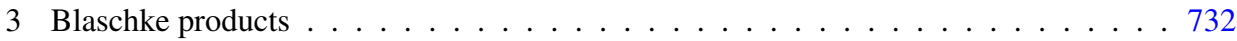

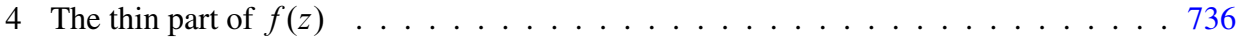

5 Bounds on repelling cycles . . . . . . . . . . . . . . . . . . . . . . 739

6 Short cycles and short geodesics . . . . . . . . . . . . . . . . . 744

7 Binding and renormalization $\ldots \ldots \ldots \ldots \ldots 74$

References . . . . . . . . . . . . . . . . . . . . . . . 748

\section{Introduction}

In this paper we show that rotation cycles on $S^{1}$ for a proper holomorphic map $f: \Delta \rightarrow \Delta$ share several of the analytic, geometric and topological features of simple closed geodesics on a compact hyperbolic surface.

Dynamics on the unit disk. Let $\Delta=\{z \in \mathbb{C}:|z|<1\}$. For $d>1$ let $\mathscr{B}_{d} \cong$ $\Delta^{(d-1)}$ denote the space of all proper holomorphic maps $f: \Delta \rightarrow \Delta$ of the form

$$
f(z)=z \prod_{1}^{d-1}\left(\frac{z-a_{i}}{1-\bar{a}_{i} z}\right),
$$

$\left|a_{i}\right|<1$. Every degree $d$ holomorphic map $g: \Delta \rightarrow \Delta$ with a fixed point in the disk can be put into the form above, by normalizing so its fixed point is $z=0$.

The maps $f \in \mathcal{B}_{d}$ have the property that $f \mid S^{1}$ is measure-preserving and $\left|f^{\prime}\right|>$ 1 on the circle. Moreover, there is a unique marking homeomorphism $\phi_{f}: S^{1} \rightarrow S^{1}$

\footnotetext{
${ }^{*}$ Research supported in part by the NSF.
} 
that varies continuously with $f$, conjugates $f$ to $p_{d}(z)=z^{d}$, and satisfies $\phi_{f}(z)=z$ when $f=p_{d}$. We define the length on $f$ of a periodic cycle $C$ for $p_{d}$ by

$$
L(C, f)=\log \left|\left(f^{q}\right)^{\prime}(z)\right|,
$$

where $q=|C|$ and $\phi_{f}(z) \in C$.

The degree of a cycle $C$ is the least $e>0$ such that $p_{d} \mid C$ extends to a covering map of the circle of degree $e$. We say $C$ is simple if $\operatorname{deg}\left(p_{d} \mid C\right)=1$; equivalently, if $p_{d} \mid C$ preserves its cyclic ordering. A finite collection of cycles $C_{i}$ is binding if $\operatorname{deg}\left(\bigcup C_{i}\right)=d$ and if $\bigcup C_{i}$ is not renormalizable (\$7).

In this paper we establish four main results.

Theorem 1.1. Any cycle with $L(C, f)<\log 2$ is simple. All such cycles $C_{i}$ have the same rotation number, and $p_{d} \mid \cup C_{i}$ preserves the cyclic ordering of $\bigcup C_{i}$.

Theorem 1.2. Every $f \in \mathscr{B}_{d}$ has a simple cycle $C$ with $L(C, f)=O(d)$.

Theorem 1.3. Let $\left(C_{i}\right)_{1}^{n}$ be a binding collection of cycles. Then for any $M>0$, the set of $f \in \mathscr{B}_{d}$ with $\sum_{1}^{n} L\left(C_{i}, f\right) \leq M$ has compact closure in the moduli space of all rational maps of degree $d$.

Theorem 1.4. The closure $E \subset S^{1}$ of the simple cycles for a given $f \in \mathscr{B}_{d}$ has Hausdorff dimension zero.

See Theorems 4.1, 5.8, 7.1 and 2.2 below.

Hyperbolic surfaces. The results above echo the following fundamental facts about compact hyperbolic surfaces $X$ of genus $g>1$ :

(1) The closed geodesics on $X$ of length less than $\log (3+2 \sqrt{2})$ are simple and disjoint.

(2) There exists a simple closed geodesic on $X$ with length $O(\log g)$.

(3) If $\left(\gamma_{i}\right)_{1}^{n}$ is a binding collection of closed curves, then the locus in Teichmüller space $\mathcal{T}_{g}$ where $\sum L\left(\gamma_{i}, X\right) \leq M$ is compact for any $M>0 .{ }^{1}$

(4) The union of the simple geodesics on $X=\Delta / \Gamma$ is a closed set of Hausdorff dimension one.

See [Bus, §4, §5], [Ker, Lemma 3.1] and [BS] for proofs. Thus simple cycles behave in many ways like simple closed geodesics.

\footnotetext{
${ }^{1}$ A collection of closed curves is binding if their geodesic representatives cut $X$ into disks.
} 
Rotation numbers and slopes. Next we formulate a more direct connection between short cycles and short geodesics. Suppose $f \in B_{d}$ satisfies $\alpha=f^{\prime}(0)=$ $\exp (2 \pi i \tau) \neq 0$. The action of $\langle f\rangle$ on $\Delta$ (with the orbit of $z=0$ removed) determines a natural quotient torus, isomorphic to

$$
X_{\tau}=\mathbb{C} /(\mathbb{Z} \oplus \mathbb{Z} \tau) \cong \mathbb{C}^{*} / \alpha^{\mathbb{Z}} .
$$

Let $L\left(p / q, X_{\tau}\right)$ denote the length of a closed geodesic on $X_{\tau}$ in the homotopy class $(-p, q)$, for the flat metric of area one. The slope $p / q \bmod 1$ which minimizes $L\left(p / q, X_{\tau}\right)$ depends only on $f^{\prime}(0) \in \Delta^{*}$. The regions $T(p / q) \subset \Delta^{*}$ where a given slope is shortest rest on the corresponding roots of unity, and form a tiling of $\Delta^{*}$ (see Figure 1).

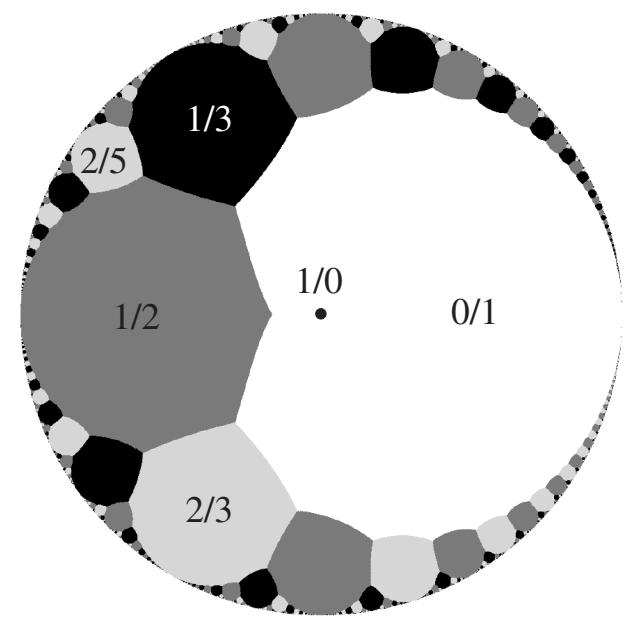

Figure 1. Tiling of $\Delta^{*}$ according to the slope of the shortest loop on the torus $\mathbb{C}^{*} / \alpha^{\mathbb{Z}}$.

In $§ 6$ we will show:

Theorem 1.5. For any $f \in \mathcal{B}_{d}$ with $f^{\prime}(0) \in T(p / q)$, there is a nonempty collection of compatible simple cycles $C_{i}$ with rotation number $p / q$ such that

$$
\frac{1}{L\left(p / q, X_{\tau}\right)^{2}} \leq \sum \frac{\pi}{L\left(C_{i}, f\right)} \leq \frac{1}{L\left(p / q, X_{\tau}\right)^{2}}+O(d),
$$

and all other cycles satisfy $L(C, f)>\epsilon_{d}>0$.

(Compatibility is defined in §2.) This result implies Theorem 1.2 and gives an alternate proof of Theorem 1.1 (with $\log 2$ replaced by $\epsilon_{d}$ ); it also yields: 
Corollary 1.6. If a sequence $f_{n} \in \mathscr{B}_{d}$ satisfies $L\left(C, f_{n}\right) \rightarrow 0$, then $f_{n}^{\prime}(0) \rightarrow$ $\exp (2 \pi i p / q)$ where $p / q$ is the rotation number of $C$.

On the other hand, we will see in $\S 3$ :

Proposition 1.7. If $f_{n} \in \mathscr{B}_{d}$ and $f_{n}^{\prime}(0) \rightarrow \exp (2 \pi i \theta)$ where $\theta$ is irrational, then $L\left(C, f_{n}\right) \rightarrow \infty$ for every cycle $C$.

Thus the cycles of moderate length guaranteed by Theorem 1.2 may be forced to have very large periods.

Petals. The proof of Theorem 1.5 is illustrated in Figure 2. Consider a map $f \in \mathscr{B}_{2}$ with $f^{\prime}(0)=\exp (2 \pi i \tau) \in T(1 / 3), \tau=1 / 3+i / 10$. The dark petals shown in the figure form the preimage $\tilde{A} \subset \Delta$ of an annulus $A$ in the homotopy class [ $3 \tau-1]$ on the quotient torus for the attracting fixed point at $z=0$. Any two adjacent rectangles within a petal give a fundamental domain for the action of $f$. The three largest petals join $z=0$ to the repelling cycle on $S^{1}$ labeled by $C=(1 / 7,2 / 7,4 / 7)$. Thus a copy of $A$ embeds in the quotient torus for the repelling cycle as well; by the method of extremal length (§5), this gives an upper bound for $L(C, f)$ in terms of $L\left(1 / 3, X_{\tau}\right)$. (The lower bound comes from the holomorphic Lefschetz fixed-point theorem.)

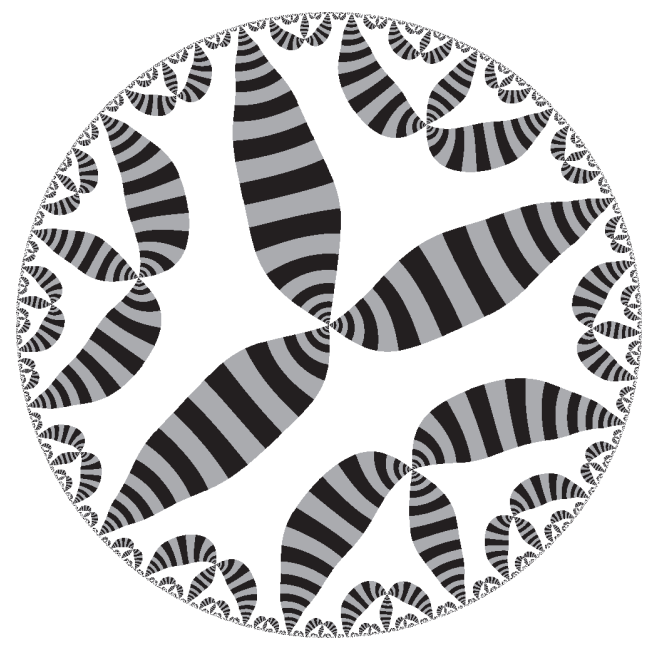

Figure 2. Petals joining $z=0$ to the $(1,2,4) / 7$ cycle on $S^{1}$.

Rational maps. Here is a related result from $\S 5$ for general rational maps $f: \widehat{\mathbb{C}} \rightarrow$ $\widehat{\mathbb{C}}$. Let $L(f)=\inf \log |\beta|$, where $\beta$ ranges over the multipliers of all repelling and indifferent periodic cycles for $f$. 
Theorem 1.8. If $f_{n} \in \mathrm{Rat}_{d}$ and $L\left(f_{n}\right) \rightarrow \infty$, then the maps $f_{n}$ have fixed points $z_{n}$ with $f^{\prime}\left(z_{n}\right) \rightarrow 0$.

Questions. We conclude with some natural questions suggested by the analogy with hyperbolic surfaces.

(1) Let $C$ be a simple cycle. Is the function $L(C, f)$ free of critical points in $\mathscr{B}_{d}$ ?

(2) Let $\left(C_{i}\right)$ be a binding collection of cycles. Does $\sum L\left(C_{i}, f\right)$ achieve its minimum at a unique point $f \in \mathscr{B}_{d}$ ?

(3) Let $\mathcal{Q} \mathscr{B}_{d}$ denote the space rational maps of the form

$$
f(z)=z \prod_{1}^{d-1}\left(\frac{z-a_{i}}{1-b_{i} z}\right)
$$

such that $\prod\left|a_{i}\right|<1, \prod\left|b_{i}\right|<1$, and $J(f)$ is a Jordan curve. Each $f \in \mathcal{Q}_{d}$ can be regarded as a marked quasiblaschke product, obtained by gluing together a pair of maps $f_{1}, f_{2} \in \mathscr{B}_{d}$ using their markings on $S^{1}$.

Does there exist an $\epsilon_{d}>0$ such for all $f \in Q_{B} \mathscr{B}_{d}$, all cycles of length shorter than $\epsilon_{d}$ are simple?

(4) Suppose the cycles $\left(C_{1}, C_{2}\right)$ are binding. Does the set of $f \in \mathcal{Q B}_{d}$ with $L\left(C_{1}, f_{1}\right)+L\left(C_{2}, f_{2}\right) \leq M$ have compact closure in the moduli space of all rational maps of degree $d$ ?

The analogous questions for hyperbolic surfaces and quasifuchsian groups are known to have positive answers [Ker, §3], [Ot], [Th, Theorem 4.4].

Notes and references. This paper is a sequel to [Mc4] and [Mc5] in which we construct a Weil-Petersson metric on $\mathscr{B}_{d}$ and an embedding of $\mathscr{B}_{d}$ into the space of invariant measures for $p_{d}(z)=z^{d}$.

Simple cycles in degree two play a central role in the combinatorics of the Mandelbrot set [DH], [Ke], and are studied for higher degree in [Gol] and [GM]. Extremal length arguments similar to those we use in $\$ 5$ are well-known both in the theory of Kleinian groups [Bers, Theorem 3], [Th, Proposition 1.3], [Mc1, §6.3], [Pet1], [Mil2] and rational maps [Pom], [Lev], [Hub], [Pet2]. The quotient Riemann surface of a general rational map is discussed in [McS]; other aspects of the dictionary between rational maps and Kleinian groups are presented in [Mc2]. See [PL] for a related discussion of spinning degenerations of the quotient torus.

\section{Simple cycles}

In this section we discuss the combinatorics of periodic cycles for the map $p_{d}(t)=$ $d \cdot t \bmod 1$, and prove the closure of the simple cycles has Hausdorff dimension zero. 
Degree and rotation number. Let $S^{1}=\mathbb{R} / \mathbb{Z}$. Given $a \neq b \in S^{1}$, let $[a, b] \subset S^{1}$ denote the unique subinterval that is positively oriented from $a$ to $b$. We write $a<c<b$ if $c \in[a, b]$. The length of an interval is denoted $|I|$.

Let $f: S^{1} \rightarrow S^{1}$ be a topological covering map of degree $d>0$, and suppose $f(X)=X$. The degree of $f \mid X$, denoted $\operatorname{deg}(f \mid X)$, is the least $e>0$ such that $f \mid X$ extends to a topological covering $g: S^{1} \rightarrow S^{1}$ of degree $e$.

Note that $\operatorname{deg}(f \mid X)=1$ iff $f$ preserves the cyclic ordering of $X$, in which case $f \mid X$ also has a well-defined rotation number $\rho(f \mid X) \in S^{1}$. If $X$ is finite then $\rho(f \mid X)=p / q$ is rational and the orbits of $f \mid X$ have size $q$.

Example. Suppose $X=\left\{x_{0}, x_{1}, \ldots, x_{n}=x_{0}\right\}$ in increasing cyclic order, and $f \mid X$ is a permutation; then we have

$$
\operatorname{deg}(f \mid X)=\sum_{0}^{n-1}\left|\left[f\left(x_{i}\right), f\left(x_{i+1}\right)\right]\right| .
$$

Indeed, an extension of $f \mid X$ of minimal degree is obtain by mapping $\left[x_{i}, x_{i+1}\right]$ homeomorphically to $\left[f\left(x_{i}\right), f\left(x_{i+1}\right)\right]$. The degree is thus a variant of the number of descents of a permutation (see e.g. [St, §1.3]).

The model map and its modular group. Now fix $d>1$, and let $p_{d}(t)=d$. $t$ mod 1 . Any expanding map $f: S^{1} \rightarrow S^{1}$ of degree $d$ is topologically conjugate to $p_{d}[\mathrm{Sh}]$.

The modular group $\operatorname{Mod}_{d} \subset \operatorname{Aut}\left(S^{1}\right)$ is the cyclic group of rotations generated by $t \mapsto 1 /(d-1)+t \bmod 1$; it coincides with the group of (degree one) topological automorphisms of $p_{d}$. Note that $\operatorname{Mod}_{d}$ acts transitively on the fixed points of $p_{d}$.

Simple cycles. A finite set $C \subset S^{1}$ is a cycle of degree $d$ if $p_{d} \mid C$ is a transitive permutation. As in $\S 1$, we say a cycle is simple if $\operatorname{deg}\left(p_{d} \mid C\right)=1$. Simple cycles $\left(C_{1}, \ldots, C_{m}\right)$ are compatible if $\operatorname{deg}\left(p_{d} \mid \cup C_{i}\right)=1$.

It is elementary to see:

Proposition 2.1. The simple cycles $\left(C_{1}, \ldots, C_{m}\right)$ are compatible iff they are pairwise compatible.

We let $\mathcal{C}_{d}$ denote the set of all cycles of degree $d$, and $\mathcal{C}_{d}(p / q) \subset \mathcal{C}_{d}$ the simple cycles with rotation number $p / q$.

Portraits of fixed points. The fixed-point portrait [Gol] of a simple cycle $C \in$ $\varphi_{d}(p / q)$ is the monotone increasing function

$$
\sigma:\{1, \ldots, d-2\} \rightarrow\{0,1, \ldots, q\}
$$


given by

$$
\sigma(j)=|C \cap[0, j /(d-1))| .
$$

This invariant specifies how $C$ is interleaved between the fixed points of $p_{d}$, which are all of the form $j /(d-1) \bmod 1$.

Basic properties. The following results are immediate from [Gol] (see especially Lemma 2 and Theorem 7).

(1) A simple cycle $C \in \ell_{d}(p / q)$ is uniquely determined by its fixed-point portrait $\sigma(j)$, and all possible monotone increasing functions $\sigma(j)$ arise.

(2) The number of simple cycles of degree $d$ and rotation number $p / q$ is $\left(\begin{array}{c}d+q-2 \\ q\end{array}\right)$.

(3) The number of cycles of period $q$ grows like $d^{q}$, while the number of simple cycles is $O\left(q^{d-1}\right)$; so most cycles are not simple.

(4) Cycles $C_{1}, C_{2} \in \mathcal{C}_{d}(p / q)$ are compatible iff their fixed-point portraits satisfy

$$
\sigma_{1}(j) \leq \sigma_{2}(j) \leq \sigma_{1}(j)+1
$$

for $0 \leq j \leq d-2$, or the same with $\sigma_{1}$ and $\sigma_{2}$ reversed.

(5) Every maximal collection of compatible cycles has cardinality $d-1$.

From portraits to cycles. A simple cycle $C \in \boldsymbol{\ell}_{d}$ can be reconstructed explicitly from its rotation number $p / q$ and its fixed-point portrait $\sigma$ as follows. Let $\tau$ be the 'transpose' of $\sigma$, namely the monotone function $\tau:\{0,1, \ldots, q-1\} \rightarrow\{0,1, \ldots$, $d-1$ g given by

$$
\tau(i)=|\{j: \sigma(j) \leq i\}|,
$$

and let

$$
\tau^{\prime}(i)=\tau(i)+ \begin{cases}0 & \text { if } 0 \leq i<q-p, \text { and } \\ 1 & \text { otherwise, }\end{cases}
$$

where $i$ is taken $\bmod q$. Then the periodic point given by $t=0 . \tau^{\prime}(0) \tau^{\prime}(p) \tau^{\prime}(2 p) \ldots$ in base $d$ generates $C$; indeed, $t$ is the 'first point' in the cycle $C$.

Examples. To simplify notation, let $\left(p_{1} / q, \ldots, p_{m} / q\right)=\left(p_{1}, \ldots, p_{m}\right) / q$, and let $\sigma=n_{1} \ldots n_{d-1}$ denote the function with values $\sigma(j)=n_{j}$.

Degree $d=2$. In the quadratic case, $\sigma$ is trivial and hence there is a unique simple cycle $C(p / q)$ for each possible rotation number; e.g.

$$
\begin{aligned}
& C(1 / 2)=(1,2) / 3 \\
& C(1 / 3)=(1,2,4) / 7 \\
& C(2 / 5)=(5,10,20,9,18) / 31 .
\end{aligned}
$$


The only cycle of period $\leq 4$ which is not simple is $C=(1,2,4,3) / 5$. For period 5 there are two such, namely $C$ and $-C$ where $C=(3,6,12,24,17) / 31$. Any two distinct quadratic simple cycles are incompatible.

Degree $d=3$. In the cubic case $p_{d}$ has two fixed points, 0 and $1 / 2$, and three cycles of period two, given by

$$
\begin{aligned}
& C(1 / 2,0)=(5,7) / 8, \\
& C(1 / 2,1)=(1,3) / 4
\end{aligned}
$$

and

$$
C(1 / 2,2)=(1,3) / 8 \text {. }
$$

The first and last are incompatible, while the other pairs are compatible. In general there are $q+1$ cubic simple cycles with rotation number $p / q$, whose fixed-point portraits are given by $\sigma(1)=0,1, \ldots, q$. Only the pairs with adjacent values of $\sigma(1)$ are compatible.

Degree $d=4$. In the quartic case there are six cycles in $\ell_{4}(1 / 2)$, generated by $t=p / 15$ with $p=1,2,3,6,7$ and 11 . The compatibility relation between these cycles is shown in Figure 3. The four visible triangles give the four distinct triples of compatible simple cycles with rotation number $1 / 2$. Note that the modular group $\operatorname{Mod}_{4} \cong \mathbb{Z} / 3$ acts by rotations on this diagram.

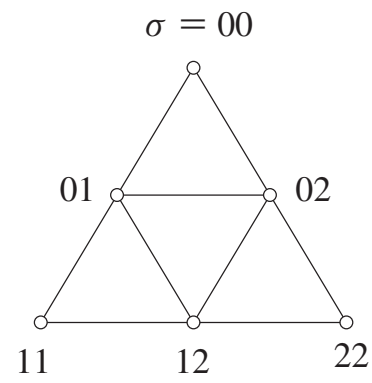

Figure 3. Compatibility of degree 4 cycles of the form $C(1 / 2, \sigma)$.

In general $\mathcal{C}_{d}(p / q)$ can be identified with the vertices of the $q$-fold barycentric subdivision of a $(d-2)$-simplex, with the top-dimensional cells corresponding to maximal collections of compatible cycles.

Sample computation in degree $d=5$. To compute $C(3 / 7,013)$, we first use equation (2.1) to compute the 'transpose' $\tau=1223333$ of $\sigma=013$. Note that the graphs of $\sigma$ and $\tau$, shown in white and black in Figure 4, fit together to form a rectangle. Evaluating $\tau^{\prime}=1223444$ along the sequence $i p \bmod q, i=0,1,2, \ldots$ we obtain the base 5 expansion $t=0 . \overline{1342424}_{5}=6966 / 19531$ for a generator of $C$. 

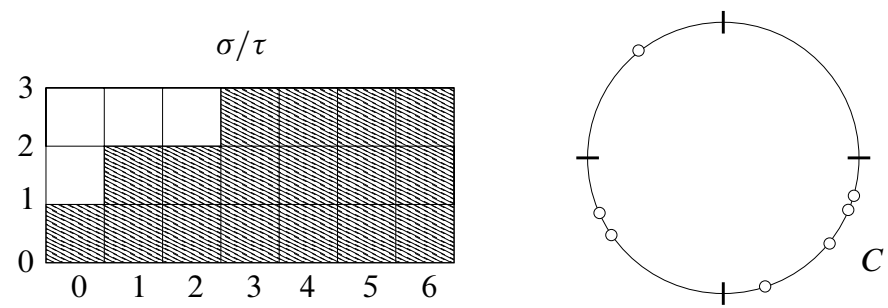

Figure 4 . The degree 5 simple cycle with rotation number $3 / 7$ and $\sigma=013$.

The cycle $C$, along with the four fixed points of $p_{5}$, is drawn at the right in Figure 4. Note that $\sigma=013$ gives the running total of the number of points of $C$ in the first three quadrants.

Comparison with simple geodesics. The simple cycles for $p_{d} \mid S^{1}$ behave in many ways like simple closed geodesics on a compact hyperbolic surface $X=\Delta / \Gamma$ of genus $g$, with compatible cycles corresponding to disjoint geodesics. For example, every maximal collection of disjoint simple closed curves on $X$ has $3 g-3$ elements, just as every maximal collection of compatible cycles for $p_{d}$ has $d-1$ elements.

It is also known that the endpoints of lifts of simple geodesics lie in a closed set $E \subset S^{1}$ of Hausdorff dimension zero [BS]. The analogous statement for simple cycles is:

Theorem 2.2. The closure $E$ of the union of all simple cycles $C \subset S^{1}$ of degree $d$ has Hausdorff dimension zero.

Proof. Let us say a finite set $P \subset S^{1}$ is a precycle if it is the forward orbit of preperiodic point $x \in S^{1}$ under $p_{d}$. We say $P$ is simple, with rotation number $p / q$, if $p_{d} \mid P$ extends to a continuous, monotone increasing map $f: S^{1} \rightarrow S^{1}$ with rotation number $p / q$. Then $q \leq n$ and the periodic part $C$ of $P$ is a simple cycle.

Let $\mathcal{P}_{d}(n, p / q)$ denote the set of all simple precycles of length $n$ and rotation number $p / q$. The argument that shows $\left|\mathcal{C}_{d}(p / q)\right|=O\left(q^{d-2}\right)$ can be adapted to show that $\left|\mathcal{P}_{d}(n, p / q)\right|=O\left(n^{d-2}\right)$ as well.

Now fix $N>0$. We claim that every $x \in E$ lies within distance $O\left(d^{-N}\right)$ of a simple precycle $P$ with $|P| \leq N$. To find this precycle, simply increase $x$ continuously until two of the points among $x, f(x), \ldots, f^{N}(x)$ coincide. This requires moving $x$ only slightly, since $\left|\left(f^{N}\right)^{\prime}(x)\right|=d^{N}$.

Thus $E$ is contained in a neighborhood of diameter $O\left(d^{-N}\right)$ of the union $E_{N}$ of all simple precycles with $|P| \leq N$. Since $\left|E_{N}\right|=O\left(N^{d+2}\right)$ grows only like a polynomial in $N$, this implies $\operatorname{dim}(E)=0$. 
Proof of Theorem 1.4. The Hölder continuous conjugacy $\phi_{f}$ between $f$ and $p_{d}$ preserves sets of Hausdorff dimension zero.

Remark: Invariant measures. The basic properties of simple cycles can also be developed using the correspondence between invariant measures and covering relations established in [Mc5]. For example, any union $D=\bigcup C_{i}$ of compatible cycles in $\ell_{d}(p / q)$ arises as the support of an invariant measure $v$ for $p_{d} \mid S^{1}$. Invariant measures, in turn, correspond bijectively to covering relations $(F, S)$ of degree $d$. In the case at hand, $F(t)=t+p / q \bmod 1$ and $S$ is a divisor on $S^{1}$ of degree $d-1$. By perturbing $S$ so its points have multiplicity one, we obtain a nearby invariant measure $v^{\prime}$ whose support $D^{\prime} \supset D$ is a maximal union of exactly $(d-1)$ compatible cycles (property (5) above).

The compactification of the space of Blaschke products by covering relations $(F, S)$ is discussed in the following section.

Question. Is there a useful notion of intersection number for a pair of cycles?

\section{Blaschke products}

This section presents basic facts about marked Blaschke products, their derivatives and their images in the moduli space of all rational maps. See [Mc5] for related background material.

Blaschke products. Identify $S^{1}=\mathbb{R} / \mathbb{Z}$ with the unit circle in the complex plane, using the coordinate $z=\exp (2 \pi i t)$. Let $\Delta=\{z:|z|<1\}$ be the unit disk, and $\Delta^{(n)}$ its $n$-fold symmetric product.

Given $d>1$, let $\mathcal{B}_{d} \cong \Delta^{(d-1)}$ denote the space of Blaschke products $f: \Delta \rightarrow \Delta$ of the form

$$
f(z)=z \prod_{1}^{d-1}\left(\frac{z-a_{i}}{1-\bar{a}_{i} z}\right)
$$

with $a_{i} \in \Delta$. Note that $f$ extends to a rational map on the whole Riemann sphere, and $f \mid S^{1}$ is a covering map of degree $d$.

A proper holomorphic map $g: \Delta \rightarrow \Delta$ of degree $d>1$ is conjugate to some $f \in \mathscr{B}_{d}$ iff $g$ has a fixed point.

Derivatives and measure. By logarithmic differentiation, any $f \in \mathscr{B}_{d}$ satisfies

$$
\left|f^{\prime}(z)\right|=1+\sum_{1}^{d-1} \frac{1-\left|a_{i}\right|^{2}}{\left|z-a_{i}\right|^{2}}
$$


for $z \in S^{1}$. In particular, $f \mid S^{1}$ is expanding.

More importantly, $f \mid S^{1}$ preserves normalized Lebesgue measure $\lambda$ on the circle; equivalently, $f_{*}(d z / z)=d z / z$, as can be verified by residue considerations. This means

$$
\sum_{f(w)=z}\left|f^{\prime}(w)\right|^{-1}=1
$$

for any $z \in S^{1}$.

Markings. All $f \in \mathscr{B}_{d}$ are topologically conjugate to the model mapping $p_{d}(z)=$ $z^{d}$. A marking for $f$ the choice of one such conjugacy, i.e. the choice of a degree one homeomorphisms $\phi: S^{1} \rightarrow S^{1}$ such that

$$
f(z)=\phi^{-1} \circ p_{d} \circ \phi(z) .
$$

There is a unique marking $\phi_{f}$ which varies continuously in $f$ and satisfies $\phi_{f}(z)=$ $z$ when $f=p_{d}$. Thus $\mathscr{B}_{d}$ can be regarded as the space of marked Blaschke products.

The modular group $\operatorname{Mod}_{d} \cong \mathbb{Z} /(d-1)$ acts on $\mathscr{B}_{d}$ by $\left(a_{i}\right) \mapsto\left(\zeta a_{i}\right)$ where $\zeta^{d-1}=1$. Its orbits correspond to different markings of the same map. Thus $f_{1}, f_{2} \in \mathscr{B}_{d}$ are conformally conjugate on $\Delta$ iff they are in the same orbit of the modular group.

Lengths. The canonical marking allows one to label the cycles of $f$ by the cycles of $p_{d}$. We define the length on $f$ of a cycle $C \in \mathcal{C}_{d}$ of period $q$ by

$$
L(C, f)=\log \left|\left(f^{q}\right)^{\prime}(z)\right|
$$

for any $z \in S^{1}$ with $\phi_{f}(z) \in C$.

Limits of lower degree. The space of Blaschke products has a natural compactification $\overline{\mathcal{B}}_{d} \cong \bar{\Delta}^{(d-1)}$, whose boundary points $\left(a_{i}\right)$ can be interpreted as pairs $(F, S)$ consisting of a Blaschke product

$$
F(z)=z \prod_{\left|a_{i}\right|<1}\left(\frac{z-a_{i}}{1-\bar{a}_{i} z}\right) \cdot \prod_{\left|a_{i}\right|=1}\left(-a_{i}\right)
$$

and a divisor of sources

$$
S=\sum_{\left|a_{i}\right|=1} 1 \cdot a_{i} \in \operatorname{Div}\left(S^{1}\right),
$$

satisfying $\operatorname{deg} F+\operatorname{deg} S=d$. It is easy to see: 
Proposition 3.1. A sequence $f_{n} \in \mathcal{B}_{d}$ converges to $(F, S) \in \partial \mathscr{B}_{d}$ iff

(i) $f_{n}(z) \rightarrow F(z)$ uniformly on compact subsets of $\widehat{\mathbb{C}}-\operatorname{supp} S$, and

(ii) the zeros $Z\left(f_{n}\right)$ converge to $Z(F)+S$ as divisors on $\widehat{\mathbb{C}}$.

More generally, the space Rat $_{d}$ of degree $d$ rational maps $f: \widehat{\mathbb{C}} \rightarrow \widehat{\mathbb{C}}$ has a compactification $\overline{\mathrm{Rat}}_{d} \cong \mathbb{P}^{2 d+1}$, whose boundary points $(F, S)$ are pairs consisting of a rational map $F$ and an effective divisor $S \in \operatorname{Div}(\widehat{\mathbb{C}})$ with $\operatorname{deg}(F)+\operatorname{deg}(S)=d$. We have $f_{n} \rightarrow(F, S)$ in $\overline{\mathrm{Rat}}_{d}$ iff their graphs satisfy

$$
\operatorname{gr}\left(f_{n}\right) \rightarrow \operatorname{gr}(F)+S \times \widehat{\mathbb{C}}
$$

as divisors of degree $(1, d)$ on $\widehat{\mathbb{C}} \times \widehat{\mathbb{C}}(\mathrm{cf} .[\mathrm{D}, \S 1])$.

Radial bounds on $f^{\prime}(z)$. The following elementary observation is useful for studying limits as above.

Proposition 3.2. For any proper holomorphic map $f: \Delta \rightarrow \Delta$ and $\zeta \in S^{1}$, we have

$$
\sup _{r \in[0,1]}\left|f^{\prime}(r \zeta)\right| \leq 4\left|f^{\prime}(\zeta)\right|
$$

Note that we do not require that $f(0)=0$. This bound is sharp, as can be seen by considering $f(z)=(z+a) /(1+a z)$ as $a \rightarrow 1-$.

Proof. We can write

$$
f(z)=e^{i \theta} \prod_{1}^{d} M_{i}(z),
$$

where $M_{i}(z)=\left(z-a_{i}\right) /\left(1-\bar{a}_{i} z\right)$ and $a_{i} \in \Delta$. Composing with a rotation, we can also assume that $\zeta=1$. For $r \in[0,1]$ we have

$$
\left|\frac{M_{i}^{\prime}(r)}{M_{i}^{\prime}(1)}\right|=\frac{\left|1-a_{i}\right|^{2}}{\left|1-r a_{i}\right|^{2}},
$$

and therefore

$$
\left|M_{i}^{\prime}(r)\right| \leq 4\left|M_{i}^{\prime}(1)\right|,
$$

since the distance from 1 to $a_{i}$ is never more than twice the distance from 1 to $r a_{i}$, Differentiating the product (3.3) and using the fact that $\left|\prod_{j \neq i} M_{j}(r)\right| \leq 1$, we obtain:

$$
\left|f^{\prime}(r)\right| \leq \sum\left|M_{i}^{\prime}(r)\right| \leq 4 \sum\left|M_{i}^{\prime}(1)\right|=4\left|f^{\prime}(1)\right| .
$$


Corollary 3.3. If $f_{n} \rightarrow(F, S) \in \overline{\mathcal{B}}_{d}, z_{n} \in S^{1}, z_{n} \rightarrow z$ and $\left|f_{n}^{\prime}\left(z_{n}\right)\right|=O(1)$, then $\lim f_{n}\left(z_{n}\right)=F(z)$.

Proof. Suppose sup $\left|f_{n}^{\prime}\left(z_{n}\right)\right|=M$; then for any $r<1$ we have

$$
\begin{aligned}
\limsup \left|f_{n}\left(z_{n}\right)-F(z)\right| & \leq \limsup \left|f_{n}\left(r z_{n}\right)-F(z)\right|+4 M(1-r) \\
& =|F(r z)-F(z)|+4 M(1-r) ;
\end{aligned}
$$

now let $r \rightarrow 1$.

Irrational rotations. As a sample application, we prove the following result stated in the Introduction:

Corollary 3.4. If $f_{n} \in \mathscr{B}_{d}$ satisfies $f_{n}^{\prime}(0) \rightarrow \exp (2 \pi i \theta)$ where $\theta$ is irrational, then $L\left(C, f_{n}\right) \rightarrow \infty$ for every cycle $C$.

Proof. Suppose to the contrary that $L\left(C, f_{n}\right)$ is bounded for some cycle $C$. Let $C_{n} \subset S^{1}$ be the corresponding periodic cycle for $f_{n}$. Pass to a subsequence such that $f_{n} \rightarrow(F, S) \in \partial \mathscr{B}_{d}$ and $C_{n} \rightarrow D \subset S^{1}$ in the Hausdorff topology. Then $F(z)=\exp (2 \pi i \theta) z$ and by Corollary 3.3 we have $F(D)=D$, contradicting the irrationality of $\theta$.

Variants. Here are two useful variants of the results above:

Proposition 3.5. For any proper holomorphic map $f: \mathbb{H} \rightarrow \mathbb{H}$ and $x \in \mathbb{R}$, we have

$$
\sup _{y}\left|f^{\prime}(x+i y)\right| \leq f^{\prime}(x) .
$$

Proposition 3.6. Assume $f_{n} \in \mathrm{Rat}_{d}$ converges to $(F, S) \in \overline{\mathrm{Rat}}_{d}, z_{n} \rightarrow z$, and $\left\|D f_{n}\left(z_{n}\right)\right\|=O(1)$ in the spherical metric on $\widehat{\mathbb{C}}$. Then we have

$$
f_{n}\left(z_{n}\right) \rightarrow F(z)
$$

provided $z_{n}$ belongs to a circle $T_{n}$ with $f_{n}^{-1}\left(T_{n}\right)=T_{n}$, and $\inf _{n} \operatorname{diam}\left(T_{n}\right)>0$.

Proofs. The first result follows directly from the representation $f(z)=a_{0} z+b_{0}+$ $\sum_{1}^{d-1} a_{i} /\left(b_{i}-z\right)$ with $a_{i}>0$ and $b_{i} \in \mathbb{R}$, and the second follows by the same argument as Corollary 3.3.

The maps $f_{n}(z)=1 /\left(1+n z^{2}\right)$ satisfy $f_{n}^{\prime}(0)=0$ and $\lim f_{n}(0)=1 \neq F(0)=$ 0 ; thus some extra hypothesis is needed to interchange limits as in Proposition 3.6. 
Moduli space of rational maps. Let MRat ${ }_{d}=\operatorname{Rat}_{d} / \operatorname{Aut}(\widehat{\mathbb{C}})$ denote the moduli space of holomorphic conjugacy classes of rational maps of degree $d>1$. A pair of Blaschke products are conjugate iff they are related by the modular group or by $z \mapsto 1 / z$; thus we have an inclusion

$$
B_{d} /\left(\operatorname{Mod}_{d} \ltimes \mathbb{Z} / 2\right) \hookrightarrow \mathrm{MRat}_{d} .
$$

The next result shows this inclusion is almost proper.

Theorem 3.7. If $f_{n} \rightarrow(F, S) \in \partial \mathcal{B}_{d}$ but $\left[f_{n}\right]$ remains bounded in MRat ${ }_{d}$, then $F(z)=z$ and $\operatorname{supp} S$ is a single point. In particular, we have $f_{n}^{\prime}(0) \rightarrow 1$.

Proof. Pass to a subsequence such $\left[f_{n}\right] \rightarrow[g] \in$ MRat $_{d}$ and $f_{n} \rightarrow(F, S) \in \partial \mathscr{B}_{d}$. Then there are conjugates $h_{n}=A_{n} f_{n} A_{n}^{-1} \rightarrow g$. Since $f_{n}$ diverges in $\mathcal{B}_{d}, A_{n} \rightarrow \infty$ in $\operatorname{Aut}(\widehat{\mathbb{C}})$. On the other hand, the measures of maximal entropy satisfy $\mu\left(h_{n}\right) \rightarrow$ $\mu(g)$ and $\mu\left(f_{n}\right) \rightarrow \mu(F, S)$, by [D, Theorem 0.1] (see also [Mc5]). Since $\mu(g)$ is nonatomic, this implies $\mu(F, S)=\lim A_{n}^{*}\left(\mu\left(h_{n}\right)\right)$ is supported at a single point. But $\operatorname{supp} \mu(F, S)$ is $F$-invariant and includes supp $S$; thus $F(z)=z$ and $\operatorname{supp} S=\{s\}$ is itself a single point.

Example. The sequence $f_{n}(z)=z\left(z+a_{n}\right) /\left(1+a_{n} z\right)$, with $a_{n}=1-1 / n$, is divergent in $\mathcal{B}_{2}$ but convergent in MRat ${ }_{2}$. To see this, normalize so the origin is a critical point instead of a fixed point; then $f_{n}(z)$ is conjugate to $h_{n}(z)=\left(z^{2}+\right.$ $\left.b_{n}\right) /\left(1+b_{n} z^{2}\right)$, and $b_{n}=a_{n} /\left(2+a_{n}\right) \rightarrow 1 / 3$ as $a_{n} \rightarrow 1$.

\section{The thin part of $f(z)$}

Let us define the thin part of $f \in \mathscr{B}_{d}$ by

$$
S_{\text {thin }}^{1}(f)=\left\{z \in S^{1}:\left|f^{\prime}(z)\right|<2\right\} .
$$

In this section we will show:

Theorem 4.1. For any $f \in \mathscr{B}_{d}$, the map $f \mid S_{\text {thin }}^{1}(f)$ extends to a degree one homeomorphism of the circle.

Corollary 4.2. All cycles of $f$ with $L(C, f)<\log 2$ are simple and compatible.

Visual angles. The derivative of

$$
f(z)=z \prod_{1}^{d-1}\left(\frac{z-a_{i}}{1-\bar{a}_{i} z}\right)
$$


can be conveniently analyzed using the hyperbolic visual angle, defined for $a, z \in \bar{\Delta}$ by

$$
\alpha(z, a)=2 \arg (z-a)-\arg (z) .
$$

This is the angle at $a$ of the hyperbolic geodesic $\overline{a z}$. For $z \in S^{1}$ we have $\arg (1-\bar{a} z)=$ $\arg (z)-\arg (z-a)$, and thus

$$
\arg (f(z))=\arg (z)+\sum_{1}^{d-1} \alpha\left(z, a_{i}\right)
$$

(Note this simplifies to $\arg (f(z))=2 \arg \left(z-a_{1}\right)$ when $d=2$.) Letting $\theta=\arg (z)$ and $\dot{\alpha}=d \alpha / d \theta$, we then obtain

$$
\left|f^{\prime}(z)\right|=1+\sum_{1}^{d-1} \dot{\alpha}\left(z, a_{i}\right)
$$

for $z \in S^{1}$.

The visual density. The visual density $\dot{\alpha}(z, a)$ is essentially the Poisson kernel; for $a=r \geq 0$ it is given by

$$
\dot{\alpha}(z, r)=\frac{1-r^{2}}{1+r^{2}-2 r \cos \theta},
$$

where $\theta=\arg z$. Geometrically, $(\dot{\alpha}(z, a) / 2 \pi) d \theta$ is the hitting measure on the circle for a random hyperbolic geodesic starting at $a$.

For fixed $z \in S^{1}$, the level sets of $\dot{\alpha}(z, a)$ are horocycles resting on $z$. Thus

$$
J(a)=\left\{z \in S^{1}: \dot{\alpha}(z, a)<1\right\}
$$

is the large arc cut off by the chord perpendicular to $\overline{0 a}$. This follows from the fact that the horocycle resting on one of the endpoints of $J(a)$ and passing through 0 also passes through $a$ (see Figure 5).

Proposition 4.3. The visual density $\dot{\alpha}(z, a) \mid J(a)$ is strictly convex, and decreases as a moves radially towards the circle. In other words, we have

$$
\dddot{\alpha}(z, a)>0 \quad \text { and }\left.\quad \frac{d}{d s} \dot{\alpha}(z, s a)\right|_{s=1}<0
$$

for all $z \in J(a)$. 


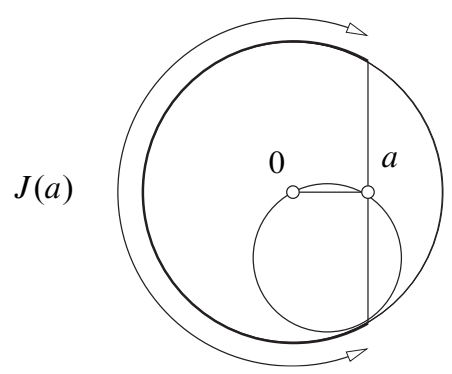

Figure 5. The arc $J(a)$ where $\dot{\alpha}(z, a)<1$.

Proof. To verify convexity, consider the case where $a=r \in[0,1)$. By (4.3), in this case we have $\dot{\alpha}=\left(1-r^{2}\right) / u$ where $u=1+r^{2}-2 r \cos \theta$. We may assume $\theta \in(0, \pi)$. Cross-multiplying and differentiating, we obtain

$$
\begin{aligned}
& \dot{\alpha} u=1-r^{2}, \\
& \ddot{\alpha} u+\dot{\alpha}(2 r \sin \theta)=0, \quad \text { and } \\
& \dddot{\alpha} u+\ddot{\alpha}(4 r \sin \theta)+\dot{\alpha}(2 r \cos \theta)=0 .
\end{aligned}
$$

Since $r, u$ and $\sin \theta$ are all positive, we have $\dot{\alpha}>0$ and $\ddot{\alpha}<0$. Comparing the last two equations, we find the sign of $\dddot{\alpha}$ is the same as the sign of the determinant

$$
D=\operatorname{det}\left(\begin{array}{cc}
2 r \sin \theta & u \\
2 r \cos \theta & 4 r \sin \theta
\end{array}\right)=8 r^{2} \sin ^{2} \theta-2 r u \cos \theta .
$$

We claim $D>0$ when $z \in J(r)$, i.e. when $u=|z-r|^{2}>1-r^{2}$. The claim is evident if $\cos \theta$ is negative, so assume $\theta \in(0, \pi / 2)$; then

$$
u=|z-r|^{2} \leq|z-1|^{2} \leq 2(\operatorname{Im} z)^{2}=2 \sin ^{2} \theta .
$$

We also have $\cos \theta=\operatorname{Re}(z)<r$ for $z \in J(r)$, and thus:

$$
D \geq 4 r^{2} u-2 r^{2} u>0 .
$$

The proof of the density decreasing property is straightforward.

Properties of the thin part of $\boldsymbol{f}$. We can now show that $f \mid S_{\text {thin }}^{1}(f)$ acts like a rotation. We first observe:

Proposition 4.4. For any $f \in \mathcal{B}_{d}$,

(i) the map $f \mid S_{\text {thin }}^{1}(f)$ is injective,

(ii) we have $S_{\text {thin }}^{1}(f) \subset \bigcap J\left(a_{i}\right)$, 
(iii) $S_{\text {thin }}^{1}(f)$ consists of at most $(d-1)$ disjoint open intervals, and

(iv) $S_{\text {thin }}^{1}(f)$ increases as the zeros $a_{i}$ of $f$ move radially towards the circle.

Proof. If $f\left(x_{1}\right)=f\left(x_{2}\right)$ for two distinct points in $S_{\text {thin }}^{1}(f)$, then $\left|f^{\prime}\left(x_{1}\right)\right|+\left|f^{\prime}\left(x_{2}\right)\right|>$ $1 / 2+1 / 2=1$, which violates the measure-preserving property (3.2) of $f$; thus $f \mid S_{\text {thin }}^{1}(f)$ is injective. Equation (4.2) implies (ii). Since $\bigcup\left(S^{1}-J\left(a_{i}\right)\right)$ has at most $(d-1)$ components, so does $I=\bigcap J\left(a_{i}\right)$. By Proposition $4.3,\left|f^{\prime}(z)\right|$ is locally convex on $I$; thus the intersection of $S_{\text {thin }}^{1}(f)$ with any component of $I$ is connected, and (iii) follows. The density decreasing property stated in Proposition 4.3 implies (iv).

Proof of Theorem 4.1. By moving the points $\left(a_{i}\right)$ radially to the circle, we obtain a smooth 1-parameter family of maps $f_{t} \in \overline{\mathcal{B}}_{d}, t \in[0,1]$, with $f_{0}=f$ and $f_{1}=(F, S)$. Since $\operatorname{deg}(S)=d-1$, we have $\operatorname{deg}(F)=1$. Proposition 4.4 implies that $f_{t} \mid T_{t}=S_{\text {thin }}^{1}\left(f_{t}\right)$ is injective, $T_{s} \subset T_{t}$ when $s<t$, and $\operatorname{supp} S \cap T_{t}=\varnothing$. Thus for any three distinct points $x_{i} \in S_{\text {thin }}^{1}(f)$, the triple $\left(f_{t}\left(x_{1}\right), f_{t}\left(x_{2}\right), f_{t}\left(x_{3}\right)\right)$ moves by isotopy as $t$ increases from 0 to 1 , and converges to $\left.F\left(x_{1}\right), F\left(x_{2}\right), F\left(x_{3}\right)\right)$ as $t \rightarrow 1$. Since $F$ is a rotation, it preserves the cyclic ordering of the points $\left(x_{i}\right)$, so the same is true of $f$. Consequently $f$ extends from $S_{\text {thin }}^{1}(f)$ to an orientation-preserving homeomorphism of the circle.

\section{Bounds on repelling cycles}

In this section we show that every $f \in \mathcal{B}_{d}$ has a simple cycle with $L(C, f)=O(d)$, and obtain related results for general rational maps.

Moduli and tori. We begin by summarizing some well-known facts about extremal length on tori.

Any point $\tau \in \mathbb{H}$ determines a complex torus

$$
X_{\tau}=\mathbb{C} /(\mathbb{Z} \oplus \mathbb{Z} \tau)
$$

with a flat metric inherited from the plane, and a distinguished basis $\langle 1, \tau\rangle$ for its fundamental group. Factoring the covering map $\mathbb{C} \rightarrow X_{\tau}$ through the map $\xi: \mathbb{C} \rightarrow$ $\mathbb{C}^{*} \cong \mathbb{C} / \mathbb{Z}$ given by $\xi(z)=\exp (2 \pi i z)$, we have

$$
X_{\tau}=\mathbb{C}^{*} / \alpha^{\mathbb{Z}}
$$

where $\alpha=\xi(\tau)$ satisfies $0<|\alpha|<1$. The same construction can be made when $-\tau \in \mathbb{H}$; then $|\alpha|>1$.

Given a slope $p / q \in \mathbb{Q} \cup\{\infty\}$, let $\gamma_{p / q} \subset X_{\tau}$ denote the simple closed geodesic obtained as the projection of the line $\mathbb{R} \cdot(\tau-p / q)$ from $\mathbb{C}$ to $X_{\tau}$. Its preimage $\tilde{\gamma}_{p / q}$ 
in the intermediate cover $\mathbb{C}^{*}$ consists of $q$ arcs joining 0 to $\infty$, cyclically permuted with rotation number $p / q$ by $z \mapsto \alpha z$.

Any annulus $A$ is conformally equivalent to a right cylinder, which is unique up to scale. The ratio $\bmod (A)=h / c$ between the height and circumference of this cylinder is the modulus of $A$.

The maximum modulus of an annulus $A \subset X_{\tau}$ homotopic to $\gamma_{p / q}$ is given by

$$
\bmod \left(p / q, X_{\tau}\right)=\frac{\operatorname{area}\left(X_{\tau}\right)}{L\left(\gamma_{p / q}, X_{\tau}\right)^{2}}=\frac{|\operatorname{Im} \tau|}{|q \tau-p|^{2}}
$$

(assuming $\operatorname{gcd}(p, q)=1)$. This maximum is realized by taking $A=X_{\tau} \backslash \gamma_{p / q}$. The set of $\tau \in \mathbb{H}$ with $\bmod \left(p / q, X_{\tau}\right) \geq m$ is a horoball of diameter $1 /\left(m q^{2}\right)$ resting on the real axis at $p / q$. For $p / q=1 / 0$ we have

$$
\bmod \left(\infty, X_{\tau}\right)=|\operatorname{Im} \tau|
$$

The intersection inequality

$$
\bmod \left(p / q, X_{\tau}\right) \bmod \left(r / s, X_{\tau}\right) \leq\left(\operatorname{det}\left(\begin{array}{ll}
p & q \\
r & s
\end{array}\right)\right)^{-2}
$$

is easily verified by considering the determinant of the lattice $\mathbb{Z}(q \tau-p) \oplus \mathbb{Z}(s \tau-r)$. This inequality implies:

There is at most one slope with $\bmod \left(p / q, X_{\tau}\right)>1$.

On the other hand we have:

Proposition 5.1. For any $\tau \in \mathbb{H}$, there exists a slope $p / q \in \mathbb{Q} \cup\{\infty\}$ such that

$$
\bmod \left(p / q, X_{\tau}\right) \geq \sqrt{3} / 2 .
$$

Proof. Since the statement is invariant under the action of $\mathrm{SL}_{2}(\mathbb{Z})$ on $\mathbb{H}$, it suffices to verify it when $\tau$ lies in the fundamental domain $|\tau| \geq 1,|\operatorname{Re} \tau| \leq 1 / 2$; and in this case, we have $\bmod \left(\infty, X_{\tau}\right)=\operatorname{Im} \tau \geq \sqrt{3} / 2$.

Rational maps. Now let $f: \widehat{\mathbb{C}} \rightarrow \widehat{\mathbb{C}}$ be a rational map of degree $d>1$. If $z \in \widehat{\mathbb{C}}$ is a point of period $q$, its multiplier is given by $\beta=\left(f^{q}\right)^{\prime}(z)$. The grand orbit of $z$ is the set $\bigcup_{i, j>0} f^{-i} \circ f^{j}(z)$.

Suppose $f$ has a fixed point at $z=0$ and a periodic point $w \neq 0$ with period $q$. We say $w$ has rotation number $p / q$ relative to $z=0$ if there are $\operatorname{arcs}\left(\delta_{i}\right)_{0}^{q-1} \subset \widehat{\mathbb{C}}$ joining $z=0$ to $f^{i}(w)$, meeting only at $z=0$, which are cyclically permuted by $f$ with rotation number $p / q$. 
Theorem 5.2. Let $f$ be a rational map with an attracting fixed-point at $z=0$, with multiplier

$$
\alpha=f^{\prime}(0)=\exp (2 \pi i \tau) \neq 0 .
$$

Let $e$ be the number of grand orbits of critical points in the immediate basin $\Omega$ of $z=0$. Then for each $p / q \in \mathbb{Q}$, there exists a repelling or parabolic periodic point $w \in \partial \Omega$ such that

(1) the rotation number of $w$ relative to $z=0$ is $p / q$; and

(2) its multiplier has the form $\beta=\left(f^{q}\right)^{\prime}(w)=\exp (-2 \pi i \sigma)$, where $\sigma=0$ or

$$
\frac{\operatorname{Im} \sigma}{|\sigma|^{2}} \geq \frac{\bmod \left(p / q, X_{\tau}\right)}{e} .
$$

In particular, we have

$$
|\beta| \leq\left(\exp \left(\frac{2 \pi}{\bmod \left(p / q, X_{\tau}\right)}\right)\right)^{e}
$$

Proof. Let $\Omega^{*}$ denote the immediate basin of $z=0$ with the grand orbits of all critical points in $\Omega$ and of $z=0$ deleted. Then $f: \Omega^{*} \rightarrow \Omega^{*}$ is a covering map. Moreover, the holomorphic linearizing map

$$
\phi(z)=\lim \alpha^{-n} f^{n}(z)
$$

is defined for all $z \in \Omega^{*}$, and satisfies $\phi(f(z))=\alpha f(z)$. Consequently $\phi$ descends to an inclusion of the space of grand orbits $Y=\Omega^{*} /\langle f\rangle$ into the torus $X_{\tau}=\mathbb{C}^{*} / \alpha^{\mathbb{Z}}$, making the diagram

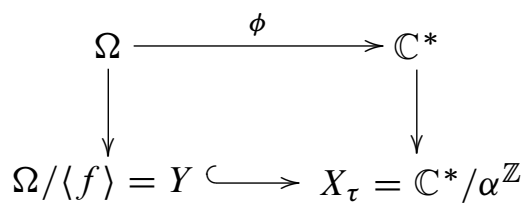

commute. By assumption we have $\left|Y-X_{\tau}\right|=e$.

For a given $p / q \in \mathbb{Q}$, the geodesics parallel to $\gamma_{p / q}$ passing through the punctures of $Y$ cut it into $\leq e$ parallel annuli, one of which satisfies

$$
\bmod (A) \geq \bmod \left(p / q, X_{\tau}\right) / e .
$$

Let $\delta \subset A$ be the core curve of $A$, and $\delta_{0} \subset \Omega^{*}$ one of its lifts which is incident to $z=0$. Let $\delta_{i}=f^{i}\left(\delta_{0}\right)$. By construction, the $\operatorname{arc} \delta_{0}$ is invariant under $f^{q}$, and $f^{q} \mid \delta_{0}$ is a bounded translation in the hyperbolic metric on $\Omega^{*}$. Consequently $\delta_{0}$ must join 
$z=0$ to another fixed point $w$ of $f^{q}$ in $\partial \Omega$. By the Snail Lemma [Mil1, Lem. 16.2], $w$ is repelling or parabolic.

We have seen that the preimage of $\gamma_{p / q}$ on $\mathbb{C}^{*}$ consists of $q$ arcs, cyclically permuted with rotation number $p / q$ by $z \mapsto \alpha z$. Since $\phi$ is a homeomorphism near $z=0$, the $\operatorname{arcs} \delta_{0}, \ldots, \delta_{q-1}$ are also cyclically permuted with rotation number $p / q$ by $f$. In particular $w$ has rotation number $p / q$ relative to $z=0$.

Now suppose $w$ is repelling, with multiplier $\beta$. Choose an injective branch of $f^{-q}$ defined on a punctured neighborhood $U^{*}$ of $w$ such that $f^{-q}: U^{*} \rightarrow U^{*}$ and

$$
Z=U^{*} /\left\langle f^{-q}\right\rangle \cong \mathbb{C}^{*} / \beta^{\mathbb{Z}}=X_{\sigma},
$$

where $\sigma=\log (\beta / 2 \pi i)$. There is a unique choice of the logarithm such that the invariant arc $\delta_{0} \cap U^{*}$ descends to a loop isotopic to $\gamma_{0}$ on $X_{\sigma}$.

By construction, $A \subset Y$ is covered by a strip $A_{0} \subset \Omega^{*}$ which retracts to $\delta_{0}$, and hence we have an inclusion

$$
A \cong A_{0} /\left\langle f^{q}\right\rangle \hookrightarrow Z \cong X_{\sigma}
$$

in the same homotopy class as $\gamma_{0}$. This implies

$$
\bmod \left(0, X_{\sigma}\right) \geq \bmod (A),
$$

and the bound (5.3) follows from equations (5.1) and (5.5).

Corollary 5.3. If $f \in \mathrm{Rat}_{d}$ has an attracting fixed point with multiplier satisfying

$$
|\alpha|>\exp (-\pi \sqrt{3})=0.0043 \ldots,
$$

then it also has a repelling or parabolic cycle with multiplier satisfying

$$
|\beta| \leq \exp (4 \pi / \sqrt{3})^{2 d-2} \leq 1416^{2 d-2} .
$$

Proof. The lower bound on $|\alpha|$ implies $\operatorname{Im}(\tau)=\bmod \left(\infty, X_{\tau}\right)<\sqrt{3} / 2$, where $\tau=(\log \alpha) / 2 \pi i$. Hence $\bmod \left(p / q, X_{\tau}\right) \geq \sqrt{3} / 2$ for some $p / q \in \mathbb{Q}$, by Proposition 5.1. Now apply equation (5.4) and note that $e \leq 2 d-2$.

Corollary 5.4. If a map $f \in \mathrm{Rat}_{d}$ has an attracting fixed point with multiplier $\alpha$, then it also has a repelling or parabolic cycle with multiplier satisfying

$$
|\beta| \leq(\exp (4 \pi / \sqrt{3}) /|\alpha|)^{2 d-2} .
$$

Proof. Choose $\tau=(\log \alpha) / 2 \pi i=x+i y$ with $x \in[-1 / 2,1 / 2]$. The previous corollary shows the desired bound holds when $y<\sqrt{3} / 2$. For $y \geq \sqrt{3} / 2$ we have

$$
m=\bmod \left(0, X_{\tau}\right)^{-1} \leq \frac{x^{2}+y^{2}}{y} \leq \frac{1}{2 \sqrt{3}}+y<\frac{2}{\sqrt{3}}+y,
$$

which implies $\exp (2 \pi / m) \leq \exp (4 \pi / \sqrt{3}) /|\alpha|$; thus by (5.4) the desired bound holds in this case as well. 
The bottom of the spectrum. Here is a qualitative consequence of the preceding corollary.

Let the spectrum $S(f) \subset \mathbb{C}$ be the set of all multipliers $\beta$ that arise from periodic points of $f \in$ Rat $_{d}$, and let

$$
L(f)=\inf \{\log |\beta|: \beta \in S(f) \text { and }|\beta| \geq 1\} .
$$

By the fixed-point formula for rational maps [Mil1, Theorem 12.4], the multipliers of $f$ at its fixed points satisfy

$$
\sum \frac{1}{\mu_{j}-1}=1,
$$

provided no $\mu_{j}=1$; in particular, $\left|\mu_{j}\right| \leq d+1$ for some $j$. Thus if $f$ has no attracting fixed points, it satisfies

$$
L(f) \leq \log (d+1)
$$

Combining this observation with Corollary 5.4, we obtain:

Corollary 5.5. Let $f_{n} \in$ Rat $_{d}$ be a sequence of rational maps with $L\left(f_{n}\right) \rightarrow \infty$. Then the maps $f_{n}$ have fixed points with multipliers $\alpha_{n} \rightarrow 0$.

Examples. It is easy to that $f_{n}(z)=z^{2}+n^{2}$ satisfies $L\left(f_{n}\right) \rightarrow \infty$ as $n \rightarrow \infty$, since its Julia set lies close to $\pm n$. Of course $f_{n}$ has a fixed point at infinity with multiplier $\alpha_{n}=0$.

Parabolics must be included in the definition of $L(f)$ to obtain Corollary 5.5. In fact, if we let $L^{*}(f)=\inf \{\log |\beta|: \beta \in S(f),|\beta|>1\}$, then $f_{n}(z)=z-1 / z+n$ satisfies $L^{*}\left(f_{n}\right) \rightarrow \infty$ even though $f_{n}$ has no attracting fixed point. (The map $f_{n}(z)$ behaves like the Hecke group $\langle z \mapsto-1 / z, z \mapsto z+n\rangle$; cf. [Mc3, Theorem 6.2].)

Question. Does Corollary 5.5 remain true if only parabolic and repelling multipliers are included in the definition of $L(f)$ ?

Blaschke products. We now return to the setting of a proper map $f: \Delta \rightarrow \Delta$ fixing $z=0$. In this case formula (5.6) implies:

Proposition 5.6. The multipliers $\left(\lambda_{i}\right)_{1}^{d-1}$ of $f \in \mathcal{B}_{d}$ at its fixed points on the circle satisfy

$$
\sum_{1}^{d-1} \frac{1}{\lambda_{i}-1}=\frac{1-|\alpha|^{2}}{|1-\alpha|^{2}}
$$

where $\alpha=f^{\prime}(0)$. 
Corollary 5.7. If $|\alpha|<1 / 2$, then $f$ has a repelling fixed point with multiplier satisfying $1<\beta \leq 1+(d-1) / 3$.

Theorem 5.8. Every $f \in B_{d}$ has a simple cycle with $L(C, f)=O(d)$.

Proof. Combine Corollaries 5.4 and 5.7.

\section{Short cycles and short geodesics}

In this section we use the fixed-point formula for rational maps to obtain the following more detailed connection between the short cycles for $f$ and the short geodesics on its quotient torus.

Theorem 6.1. Given $f \in \mathscr{B}_{d}$ with $f^{\prime}(0)=\exp (2 \pi i \tau)$, choose $p / q \in \mathbb{Q}$ to maximize $\bmod \left(p / q, X_{\tau}\right)$. Then there exist compatible simple cycles $C_{i}$ with rotation number $p / q$, such that

(1) their lengths satisfy

$$
\bmod \left(p / q, X_{\tau}\right) \leq \pi \sum L\left(C_{i}, f\right)^{-1} \leq \bmod \left(p / q, X_{\tau}\right)+O(d) ;
$$

(2) all other cycles satisfy $L(C, f)>\epsilon_{d}>0$; and

(3) for any $r>0$, the multipliers of $f^{r}$ at its repelling fixed points satisfy

$$
\frac{1}{r} \sum^{\prime} \frac{1}{\lambda_{j}-1}=O(d),
$$

where the prime indicates that fixed points in $\bigcup C_{i}$ are excluded.

In qualitative terms, the construction shows:

Corollary 6.2. All cycles with $L(C, f)<\epsilon_{d}$ arise from short geodesics on the quotient torus for $f$.

Tiling of $\boldsymbol{\Delta}^{*}$. The slope $p / q$ mod 1 appearing in the theorem above depends only on $\alpha=f^{\prime}(0) \in \Delta^{*}$. Figure 1 of the Introduction shows the regions $T(p / q) \subset \Delta^{*}$ where a given slope maximizes the value of $\bmod \left(p / q, X_{\tau}\right)=\bmod \left(p / q, \mathbb{C} / \alpha^{\mathbb{Z}}\right)$.

This picture is nothing more than the image, under the covering map $\xi: \mathbb{H} \rightarrow \Delta^{*}$ given by $\xi(\tau)=\exp (2 \pi i \tau)$, of the tiling of $\mathbb{H}$ by $\mathrm{SL}_{2}(\mathbb{Z})$ translates of the Dirichlet region

$$
F=\{\tau \in \mathbb{H}:|\tau-n| \geq 1 \forall n \in \mathbb{Z}\}
$$


for the cusp $\tau=\infty$. The tile $T(\infty)=\xi(F)$ lies in a ball of radius $\exp (-\pi \sqrt{3}) \approx$ $1 / 230$ about the origin. In this tile the short curve is $\gamma_{\infty} \subset X_{\tau}$, which lifts to a loop around $z=0$ rather than a path connecting $z=0$ to a periodic point. Thus the length of $\gamma_{\infty}$ can go to zero without any cycle getting short.

Each remaining tile $T(p / q)$ contains a horocycle $H$ resting on the root of unity $\exp (2 \pi i p / q) \in S^{1}$. Within a still smaller horocycle $H^{\prime} \subset H, \gamma_{p / q}$ becomes very short, and hence $f$ has a very short cycle with rotation number $p / q$.

Moduli and multipliers. We begin the proof of Theorem 6.1 by connecting Diophantine properties of $\alpha \in \Delta^{*}$ to lengths of geodesics on $\mathbb{C}^{*} / \alpha^{\mathbb{Z}}$.

Lemma 6.3. For any $\alpha=\exp (2 \pi i \tau) \in \Delta^{*}$ and $q>0$, we have

$$
\sup _{p} \frac{\bmod \left(p / q, X_{\tau}\right)}{\operatorname{gcd}(p, q)^{2}}=\frac{\pi}{q} \frac{1-\left|\alpha^{q}\right|^{2}}{\left|1-\alpha^{q}\right|^{2}}+O(1) .
$$

Proof. First consider the case $q=1$, and assume $\tau$ is chosen so $|\operatorname{Re} \tau| \leq 1 / 2$. Then we have $2 \pi i \tau \approx 1-\alpha$ when either side is small, and hence

$$
\sup _{p} \bmod \left(p, X_{\tau}\right)=\frac{\operatorname{Im} \tau}{|\tau|^{2}}=\pi \frac{1-|\alpha|^{2}}{|1-\alpha|^{2}}+O(1) \text {. }
$$

The general case follows using the fact that

$$
\frac{\bmod \left(p / q, X_{\tau}\right)}{\operatorname{gcd}(p, q)^{2}}=\frac{\bmod \left(p, X_{q \tau}\right)}{q} .
$$

Proof of Theorem 6.1. Choose $p$ so that $\bmod \left(p / q, X_{\tau}\right)$ is maximized. As in Theorem 5.2, by cutting the torus $X_{\tau}$ open along $e \leq d-1$ geodesics parallel to $\gamma_{p / q}$ we obtain annuli $A_{1}, \ldots, A_{e} \subset Y$ with

$$
\bmod \left(p / q, X_{\tau}\right)=\sum \bmod \left(A_{i}\right)
$$

Each annulus $A_{i}$, when lifted to the unit disk, connects $z=0$ to a simple cycle $C_{i}$ for $f$ with rotation number $p / q$ and multiplier $\beta_{i}>1$.

The lifts of the annuli $A_{i}$ are disjoint, so the cycles $C_{i}$ are compatible. Assume for the moment they are also distinct. Since two copies of $A_{i}$ embed in the quotient torus $\mathbb{C}^{*} / \beta_{i}^{\mathbb{Z}}$ (one for the inside of the disk and one for the outside), we have

$$
2 \bmod \left(A_{i}\right) \leq \frac{2 \pi}{\log \beta_{i}}=\frac{2 \pi}{L\left(C_{i}, f\right)} .
$$

The combination of these inequalities yields:

$$
\bmod \left(p / q, X_{\tau}\right) \leq \pi \sum L\left(C_{i}, f\right)^{-1}
$$


This lower bound also holds when the cycles are not distinct; then we simply have more annuli $A_{i}$ embedded in a given torus $\mathbb{C}^{*} / \beta_{j}^{\mathbb{Z}}$.

For the upper bound, let $\left(\lambda_{j}\right)$ denote the multipliers of the repelling fixed points of $f^{q}$. Note that each cycle $C_{i}$ contributes $q$ fixed points, each with multiplier $\beta_{i}$. Combining Proposition 5.6 and Lemma 6.3, we obtain:

$$
\begin{aligned}
\frac{1}{q} \sum \frac{1}{\lambda_{j}-1} & =\frac{1}{q} \sum^{\prime} \frac{1}{\lambda_{j}-1}+\sum \frac{1}{\beta_{i}-1}=\frac{1-\left|\alpha^{q}\right|^{2}}{q\left|1-\alpha^{q}\right|^{2}} \\
& =\pi^{-1} \bmod \left(p / q, X_{\tau}\right)+O(1) .
\end{aligned}
$$

(Again, the prime indicates fixed points in $\bigcup C_{i}$ are excluded.) Since the cycles $C_{i}$ are compatible, there are no more than $d-1$ of them, and hence

$$
\sum \frac{1}{\beta_{i}-1}=\sum\left(\frac{1}{\log \beta_{i}}+O(1)\right)=\left(\sum L\left(C_{i}\right)^{-1}\right)+O(d) .
$$

This yields the upper bound in (6.1); and it also implies

$$
\frac{1}{q} \sum^{\prime} \frac{1}{\lambda_{j}-1}=O(d)
$$

That is, equation (6.2) holds for $r=q$.

To obtain (6.2) for other values of $r$, recall that by (5.2) we have $\bmod \left(s / r, X_{\tau}\right)<1$ whenever $s / r \neq p / q$. Thus if $q$ does not divide $r$, Lemma 6.3 implies

$$
\frac{1}{r} \sum^{\prime}\left(\lambda_{j}-1\right)^{-1} \leq \frac{1-\left|\alpha^{r}\right|^{2}}{r\left|1-\alpha^{r}\right|^{2}} \leq \sup _{s} \bmod \left(s / r, X_{\tau}\right)+O(1)=O(1) ;
$$

while for $r=n q$ we obtain

$$
\frac{1}{r} \sum^{\prime} \frac{1}{\lambda_{j}-1}+\frac{q}{r} \sum \frac{1}{\beta_{i}^{n}-1}=\frac{1-\left|\alpha^{r}\right|^{2}}{r\left|1-\alpha^{r}\right|^{2}}=\frac{\bmod \left(p / q, X_{\tau}\right)}{\pi n^{2}}+O(1),
$$

which again implies (6.2), since (6.1) gives

$$
\frac{q}{r} \sum \frac{1}{\beta_{i}^{n}-1}=\frac{1}{n}\left(\sum \frac{1}{n L\left(C_{i}, f\right)}+O(1)\right)=\frac{\bmod \left(p / q, X_{\tau}\right)}{\pi n^{2}}+O(d) .
$$

Finally note that equation (6.2) implies $L(C, f)>\epsilon_{d} \asymp 1 / d>0$, since any cycle $C$ of period $r$ and multiplier $\beta$, not among the $C_{i}$, contributes $1 /(\beta-1)$ to the $\operatorname{sum}(1 / r) \sum^{\prime}\left(\lambda_{j}-1\right)^{-1}$. 


\section{Binding and renormalization}

We conclude by proving the following compactness result.

Theorem 7.1. Let $\left(C_{i}\right)_{1}^{n}$ be a binding set of cycles of degree $d$. Then for any $M>0$, the set of $f \in \mathscr{B}_{d}$ such that $\sum_{i} L\left(C_{i}, f\right) \leq M$ has compact closure in MRat . $_{\text {. }}$

Corollary 7.2. The set of $f \in \mathcal{B}_{d}$ such that $\sum_{i} L\left(C_{i}, f\right) \leq M$ and $\left|f^{\prime}(0)-1\right| \geq$ $1 / M$ is compact.

Proof. By Theorem 3.7, the only way a sequence $f_{n}$ can diverge in $\mathcal{B}_{d}$ but remain bounded in MRat $d$ is if $f_{n}^{\prime}(0) \rightarrow 1$.

Definitions. Sets $A, B \subset S^{1}$ are unlinked if they lie in disjoint connected sets; equivalently, if their convex hulls in the unit disk are disjoint. A map $f: X \rightarrow X$ with $X \subset S^{1}$ is renormalizable if there is a nontrivial partition of $X$ into disjoint, unlinked subsets $X_{1}, \ldots, X_{n}$, such that every $f\left(X_{i}\right)$ lies in some $X_{j}$.

We say a collection of degree $d$ cycles $C_{1}, \ldots, C_{m}$ is binding if $\operatorname{deg}\left(p_{d} \mid \cup C_{i}\right)=$ $d$ and $p_{d} \mid \cup C_{i}$ is not renormalizable.

Proof of Theorem 7.1. Suppose to the contrary that we have a sequence $f_{n} \in \mathcal{B}_{d}$ with $\sum_{i} L\left(C_{i}, f_{n}\right) \leq M$ that is divergent in moduli space. Let

$$
D_{n}=\phi_{f_{n}}^{-1}\left(\bigcup C_{i}\right) \subset S^{1}
$$

be the finite $f_{n}$-invariant set corresponding to the binding cycles. Since $f_{n} \mid S^{1}$ is expanding, we have $\left|f_{n}^{\prime}\right| \leq e^{M}$ on $D_{n}$.

Next we conjugate the entire picture by an affine transformation depending on $n$, so that $0 \in D_{n}$ and $\operatorname{diam}\left(D_{n}\right)=1$. Then $S^{1}$ goes over to a circle $T_{n} \supset D_{n}$ invariant by $f_{n}$, and we still have $\left|f_{n}^{\prime}\right| D_{n} \mid \leq e^{M}$.

Pass to a subsequence such that $f_{n} \rightarrow(F, S) \in \overline{\text { Rat }}_{d}$. Since $f_{n}$ diverges in MRat $_{d}$, we have $\operatorname{deg}(F)<d$. Passing to a further subsequence, we can find a finite set $D$ containing zero and a circle $T \subset \widehat{\mathbb{C}}$ such that $D_{n} \rightarrow D$ and $T_{n} \rightarrow T$ in the Hausdorff topology. Note that $|D|>1$ since diam $D=1$.

By Proposition 3.6, the map $f_{n} \mid D_{n}$ converges to $F \mid D$. But if $|D|=\left|\bigcup C_{i}\right|$, the map $F \mid(D \subset T)$ is combinatorially the same as $p_{d} \mid\left(\bigcup C_{i} \subset S^{1}\right)$, contradicting our assumption that $\operatorname{deg}\left(p_{d} \mid \cup C_{i}\right)=d$. Similarly, if $|D|<\left|\cup C_{i}\right|$, then the collapse of $D_{n}$ to $D$ provides an invariant partition for $\bigcup C_{i}$, contradicting our assumption that $p_{d} \mid \cup C_{i}$ is not renormalizable. 
Examples. The single cycle $C=(3,6,12,24,17) / 31$ in degree 2 is already binding, as is any cycle of prime order with $\operatorname{deg}\left(p_{d} \mid C\right)=d$.

The first renormalizable cycle in degree 2 is $C=(1,2,4,3) / 5$. Although $\operatorname{deg}\left(p_{2} \mid C\right)=2, L\left(C, f_{n}\right)$ remains bounded as $f_{n} \in \mathscr{B}_{2}$ diverges along the sequence specified by $f_{n}^{\prime}(0)=-1+1 / n$. Indeed, $f_{n}^{2}$ can be renormalized so that $C$ converges to the cycle of period 2 for $G(z)=z-1 / z$ [Ep]; and thus $L\left(C, f_{n}\right) \rightarrow \log 9$. For more details, see [Mc6, §14].

\section{References}

[Bers] L. Bers, On boundaries of Teichmüller spaces and on Kleinian groups. I. Ann. of Math. 91 (1970), 570-600. Zbl 0197.06001 MR 0297992

[BS] J. S. Birman and C. Series, Geodesics with bounded intersection number on surfaces are sparsely distributed. Topology 24 (1985), 217-225. Zbl 0568.57006 MR 0793185

[Bus] P. Buser, Geometry and Spectra of Compact Riemann Surfaces. Progr. Math. 106, Birkhäuser, Boston, Mass., 1992. Zbl 0770.53001 MR 1183224

[D] L. DeMarco, Iteration at the boundary of the space of rational maps. Duke Math. J. 130 (2005), 169-197. Zbl 05004325 MR 2176550 MR 0762431 MR 0812271

[DH] A. Douady and J. Hubbard, Étude dynamique des polynômes complexes. Pub. Math. d'Orsay 84-2, 85-4, Université de Paris-Sud, Département de Mathématiques, Orsay, 1984, 1985. Zbl 0552.30018 Zbl 0571.30026 MR 0762431 MR 0812271

[Ep] A. L. Epstein, Bounded hyperbolic components of quadratic rational maps. Ergodic Theory Dynam. Systems 20 (2000), 727-748. Zbl 0963.37041 MR 1764925

[Gol] L. Goldberg, Fixed points of polynomial maps I. Rotation subsets of the circles. Ann. Sci. École Norm. Sup. 25 (1992), 679-685. Zbl 0771.30027 MR 1198093

[GM] L. R. Goldberg and J. Milnor, Fixed points of polynomial maps II: Fixed point portraits. Ann. Sci. École Norm. Sup. 26 (1993), 51-98. Zbl 0771.30028 MR 1209913

[Hub] J. H. Hubbard, Local connectivity of Julia sets and bifurcation loci: three theorems of J.-C. Yoccoz. In Topological Methods in Modern Mathematics, ed. by L. R. Goldberg and A. V. Phillips, Publish or Perish, Inc., 1993, 467-511. Zbl 0797.58049 MR 1215974

[Ke] K. Keller, Invariant Factors, Julia Equivalences and the (Abstract) Mandelbrot Set, Lecture Notes in Math. 1732, Springer-Verlag, Berlin 2000. Zbl 0970.37032 MR 1761576

[Ker] S. Kerckhoff, The Nielsen realization problem. Ann. of Math. 177 (1983), 235-265. Zbl 0528.57008 MR 0690845

[Lev] G. M. Levin, On Pommerenke's inequality for the eigenvalues of fixed points. Colloq. Math. 62 (1991), 167-177. Zbl 0742.30026 MR 1114630

[Mc1] C. McMullen, Iteration on Teichmüller space. Invent. Math. 99 (1990), 425-454. Zbl 0695.57012 MR 1031909

[Mc2] C. McMullen, The classification of conformal dynamical systems. In Current Developments in Mathematics, 1995, International Press, Cambridge, Mass., 1995, 323-360. Zbl 0908.30028 MR 1474980 
[Mc3] C. McMullen, Hausdorff dimension and conformal dynamics III: Computation of dimension. Amer. J. Math. 120 (1998), 691-721. Zbl 0953.30026 MR 1637951

[Mc4] C. McMullen, Thermodynamics, dimension and the Weil-Petersson metric. Invent. Math. 173 (2008), 365-425. Zbl 1156.30035 MR 2415311

[Mc5] C. McMullen, A compactification of the space of expanding maps on the circle. Geom. Funct. Anal. 18 (2008), 2101-2119. Zbl 05603850 MR 2491699

[Mc6] C. McMullen, Ribbon $\mathbb{R}$-trees and holomorphic dynamics on the unit disk. J. Topol. 2 (2009), 23-76. Zbl 05549240 MR 2499437

[McS] C. McMullen and D. Sullivan, Quasiconformal homeomorphisms and dynamics III: The Teichmüller space of a holomorphic dynamical system. Adv. Math. 135 (1998), 351-395. Zbl 0926.30028 MR 1620850

[Mil1] J. Milnor, Dynamics in One Complex Variable: Introductory Lectures. Vieweg, Wiesbaden 1999. Zbl 0946.30013 MR 1721240

[Mil2] J. Milnor, Local connectivity of Julia sets: expository lectures. In The Mandelbrot Set, Theme and Variations, ed. by Tan Lei, Cambridge University Press, Cambridge 2000, 67-116. Zbl 1107.37305 MR 1765085

[Ot] J.-P. Otal, Sur le nouage des géodésiques dans les variétés hyperboliques. C. R. Acad. Sci. Paris Sér. I Math. 320 (1995), 847-852. Zbl 0840.57008 MR 1326694

[Pet1] C. L. Petersen, A PLY inequality for Kleinian groups. Ann. Acad. Sci. Fenn. 18 (1993), 23-29. Zbl 0783.30033 MR 1207892

[Pet2] C. L. Petersen, No elliptic limits for quadratic maps. Ergodic Theory Dynam. Systems 19 (1999), 127-141. Zbl 0921.30019 MR 1676926

[PL] K. Pilgrim and T. Lei, Spinning deformations of rational maps. Conform. Geom. Dyn. 8 (2004), 52-86. Zbl 1084.37039 MR 2060378

[Pom] Ch. Pommerenke, On conformal mapping and iteration of rational functions. Complex Variables Theory Appl. 5 (1986), 117-126. MR 0846481

[Sh] M. Shub, Endomorphisms of compact differentiable manifolds. Amer. J. Math. 91 (1969), 175-199. Zbl 0201.56305 MR 0240824

[St] R. P. Stanley, Enumerative Combinatorics. Vol. I, Wadsworth Brooks/Cole Math. Ser., Wadsworth \& Brooks, Monterey, CA, 1986. Zbl 0608.05001 MR 0847717

[Th] W. P. Thurston, Hyperbolic structures on 3-manifolds II: Surface groups and 3-manifolds which fiber over the circle. Preprint, 1986.

Received August 12, 2007

Curtis T. McMullen, Mathematics Department, Harvard University, 1 Oxford St, Cambridge, MA 02138-2901, U.S.A. 